\title{
Osmotic stress and water stress have opposite effects on putrescine and proline production in excised rice leaves
}

\author{
Chien Teh Chen \& Ching Huei Kao* \\ Department of Agronomy, National Taiwan University, Taipei, Taiwan, Republic of China \\ *Author for correspondence
}

Received 24 December 1992; accepted 20 February 1993

Key words: abscisic acid, jasmonic acid methyl ester, Oryza sativa, osmotic stress, proline, putrescine, water stress

\begin{abstract}
The effects of water stress and osmotic stress (sorbitol treatment) on the production of putrescine and proline in excised rice leaves were compared. Osmotic stress and water stress were found to affect differentially the levels of putrescine and proline in excised rice leaves. Putrescine accumulation is induced by osmotic stress, whereas proline accumulation is induced by water stress. The effects of ABA on the levels of proline and putrescine are similar to those of water stress, whereas the effects of jasmonic acid methyl ester (JA-Me) are similar to those of osmotic stress. Water stress results in an increase of endogenous ABA is excised rice leaves. However, neither osmotic stress nor JA-Me has effect on endogenous ABA levels in excised rice leaves. Of particular interest is the finding that proline levels increase when putrescine levels induced by osmotic stress or JA-Me are reduced by $\mathrm{D}$-arginine and $\alpha$-methylornithine. L-arginine and L-ornithine applied exogenously also cause an increase in proline levels. It seems that L-arginine and L-ornithine are preferentially utilized as precursors for putrescine accumulation in excised rice leaves treated with osmotic stress and JA-Me, and for proline accumulation in excised rice leaves exposed to water stress and ABA.
\end{abstract}

Abbreviations: $A \mathrm{BA}=$ abscisic acid; $\mathrm{BSA}=$ bovine serum albumin; ELISA $=$ enzyme-linked immunosorbent assay; HPLC $=$ high performance chromatography; JA-Me $=$ jasmonic acid methyl ester; PVP = poly-vinylpyrrolidone

\section{Introduction}

Recently, considerable interest has been shown in the changes that take place in polyamine levels in the leaves subjected to various kinds of environmental stress. Water stress has been shown to increase the level of putrescine in leaf tissues [11, 21]. Putrescine accumulation also occurs in cereal leaf segments exposed to osmotic stress $[8,9,10$, 22]. It has long been known that proline accumulates in leaves of many plants in response to water stress [1]. Proline accumulation has also been observed in tobacco callus exposed to osmotic stress [6]. In experiments where osmotic stress is imposed in plant tissue by use of sorbitol or manni- tol, it is difficult to separate purely osmotic effects from effects due to water stress generated during such treatments. The present investigation was undertaken to compare the effects of water stress and osmotic stress (sorbitol treatment) on the accumulation of putrescine and proline in excised rice leaves.

Proline accumulation can be markedly induced by $A B A$ in excised leaves of several plant species $[5,17]$. However, ABA did not affect the levels of putrescine in detached barley leaves [17]. Thus it was of great interest to understand whether the levels of putrescine and proline in excised rice leaves treated with ABA are similar to those exposed to water stress or osmotic stress. Jasmo- 
nates and ABA show many and remarkable similarities - structurally, functionally, and regulatory [16]. Thus, the effects of jasmonic acid methyl ester (JA-Me) on the accumulation of proline and putrescine were also investigated.

\section{Materials and methods}

\subsection{Plant material and incubation condition}

Rice (Oryza sativa cv Taichung Native 1) was cultured as previously described [12]. The apical $3 \mathrm{~cm}$ segments excised from the third leaves of 12 day-old seedlings were used. A group of 10 segments was floated in a Petri dish containing $10 \mathrm{ml}$ of test solutions. Incubation was carried out at $27^{\circ} \mathrm{C}$ in the light $\left(16.7 \mu \mathrm{mol}\right.$ quanta $\left.\mathrm{m}^{-2} \mathrm{~s}^{-1}\right)$. Water stress was applied by a previously described method [3]. Briefly, leaf segments were exposed to the vapor above a solution of $0.5 \mathrm{M} \mathrm{NaCl}$. Water stress was judged by loss of fresh weight. The fresh weight loss was about 9,15 and $20 \%$ at 4,8 and $12 \mathrm{~h}$ after the experiment, respectively. Osmotic stress was achieved by floating leaf segments on $0.6 \mathrm{M}$ sorbitol solution. The fresh weight loss in osmotically stressed leaf segments was about 3,5 and $10 \%$ at 4,8 and $12 \mathrm{~h}$ after the experiment, respectively. For ABA and JA-Me treatments, the optimum concentration $(45 \mu \mathrm{M})$ to induce proline or putrescine accumulation was used.

\subsection{Determination of proline}

Proline was extracted and its concentration determined by the method of Bates et al. [2]. Leaf segments were homogenized with $3 \%$ sulfosalicylic acid and the homogenate was centrifuged. The supernatant was treated with acetic acid and acid ninhydrin, boiled for $1 \mathrm{~h}$, and then absorbance at $520 \mathrm{~nm}$ was determined. Levels of proline are expressed as $\mu \mathrm{mol} \mathrm{g}^{-1}$ initial fresh weight.

\subsection{Determination of free putrescine}

For putrescine determination, leaf segments were homogenized in 5\% perchloric acid. Putrescine levels were determined using HPLC after benzoylation as described previously [4]. Levels of putrescine are expressed as $n m o l g^{-1}$ initial fresh weight.

\subsection{Determination of $A B A$}

For ABA extraction, leaf samples were homogenized with extraction solution $(80 \%$ methanol containing $100 \mathrm{mg}^{-1}$ butylated hydroxytoluene). Extracts were filtered through Whatman No. 1 filter paper and rinsed twice with extraction solution. Filtrate was reduced to dryness in vacuo at $30^{\circ} \mathrm{C}$. Samples were resuspended in $100 \%$ methanol. $\left(\mathrm{NH}_{4}\right)_{2} \mathrm{HPO}_{4}(500 \mathrm{mM})$ was then added and the samples were allowed to stand for $10 \mathrm{~min}$ at $4{ }^{\circ} \mathrm{C}$ until ammonium salts formed. Pigments and phenolics in ammonium salt solution were removed by passing through a PVP column [14]. The combined PVP column-filtrated solutions were adjusted to $\mathrm{pH} 3.0$ with acetic acid. The acidified solution was eluted through a $\mathrm{C} 18$ cartridge. Methanol (20\%, pH 3.2) was passed through the C18 cartridge to remove polar compounds. ABA trapped in the $\mathrm{C} 18$ cartridge was then eluted with $65 \%$ ethanol ( $\mathrm{pH} 3.2$ ). ABA solution was reduced to dryness in vacuo, resuspended in Tris-buffered saline $(50 \mathrm{~m} M$ Tris $\mathrm{HCl}, 10 \mathrm{~m} M \mathrm{NaCl}, 1 \mathrm{~m} M$ $\left.\mathrm{MgCl}_{2}, 15 \mathrm{mMNaN}_{3}, \mathrm{pH} 7.5\right)$ and then subjected to HPLC. Samples were injected into a Waters M-6 UK Universal Liquid Chromatograph. They were eluted through a $250-\times 4 \mathrm{~mm} \mathrm{C18}$ reversephase column ( $5 \mu \mathrm{m}$ particle size) at a flow rate of $1 \mathrm{ml} \mathrm{min}^{-1}$ with $40 \%$ methanol in $0.02 \%$ acetic acid. ABA was collected by a fraction collector. The ABA fractions were then dried in vacuo. Samples were resuspended in Tris-buffered saline and $100 \%$ methanol and stored at $-20^{\circ} \mathrm{C}$ for ELISA analysis. ABA was quantitated by indirect ELISA according to Walker-Simmons [23] and Norman et al. [15]. ABA-4'-BSA conjugate was prepared according to Weiler [25] and used to determine ABA. ABA levels are expressed as pmol $\mathrm{g}^{-1}$ initial fresh weight.

\section{Results}

Figure 1 shows the changes with time in levels of putrescine and proline in excised rice leaves incubated in water or exposed to osmotic stress or water stress in the light. Osmotic stress markedly increases putrescine levels. However, only a slight increase in putrescine levels was observed in excised leaves exposed to water stress. At variance 


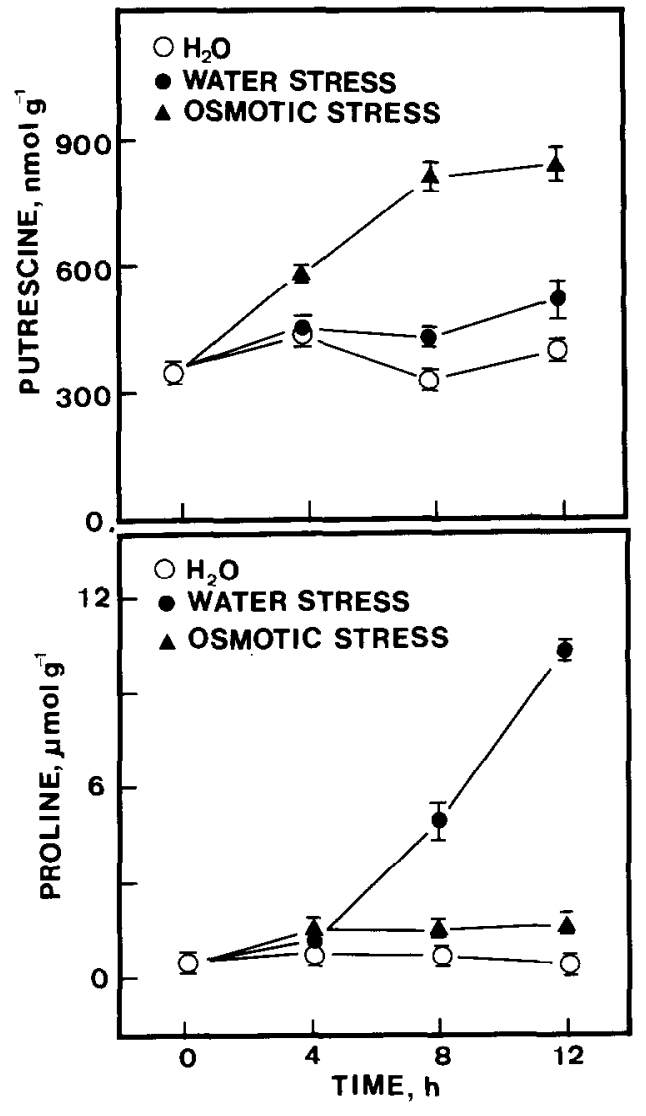

Fig. 1. Changes with time in the levels of putrescine and proline in excised rice leaves treated with water stress and osmotic stress. Excised rice leaves were treated with either water, water stress or $0.6 \mathrm{M}$ sorbitol in the light. The levels of putrescine were quantitated at the times indicated. Bars represent SE; 3 replicates.

with the results of putrescine accumulation, water stress markedly increased proline levels, but osmotic stress only caused a slight increase in proline levels. The effects of ABA and JA-Me on the accumulation of proline and putrescine are shown in Figure 2. The increase of putrescine by JA-Me was evident at $4 \mathrm{~h}$ after incubation in the light. However, ABA treatment did not result in an increase of putrescine levels. Proline levels, on the other hand, increased significantly in excised rice leaves treated with ABA, but increased slightly in those trcated with JA-Me. Clcarly, the effccts of ABA on the levels of proline and putrescine are similar to those of water stress, whereas the effects of JA-Me are similar to those of osmotic stress.

The levels of endogenous ABA rise markedly in response to water stress in several plant species

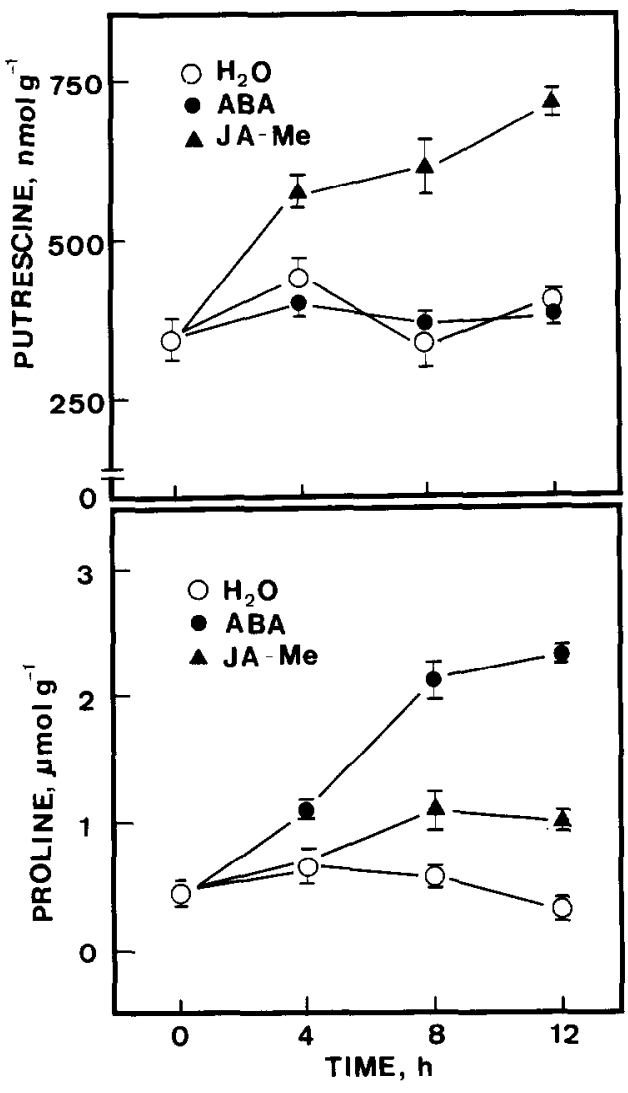

Fig. 2. Changes with time in the levels of putrescine and proline in excised rice leaves treated with ABA and jasmonic acid methyl ester (JA-Me). Excised rice leaves were treated with either water, $45 \mu M \mathrm{ABA}$ or $45 \mu M \mathrm{JA}-\mathrm{Me}$ in the light. The levels of putrescine were quantitated at the times indicated. Bars represent SE; 3 replicates.

[24]. Since exogenously applied ABA mimics the effects of water stress on the levels of proline and putrescine, it is expected that water stress may result in an increase in ABA levels in excised rice leaves. As indicated in Figure 3, the levels of $\mathrm{ABA}$, indeed, increased by water stress at $8 \mathrm{~h}$ after the start of the incubation. Neither JA-Me nor osmotic stress had any effect on the levels of ABA in excised rice leaves.

To characterize further the effects of osmotic stress and JA-Me on the accumulation of putrescine, inhibitors of the biosynthesis of putrescine, such as $\mathrm{D}$-arginine and $\alpha$-methylornithine, were applied to sorbitol- and JA-Me-treated excised rice leaves. The results are shown in Table $1 . \mathrm{D}$ arginine but not $\alpha$-methylornithine significantly reduced the levels of putrescine in excised rice 


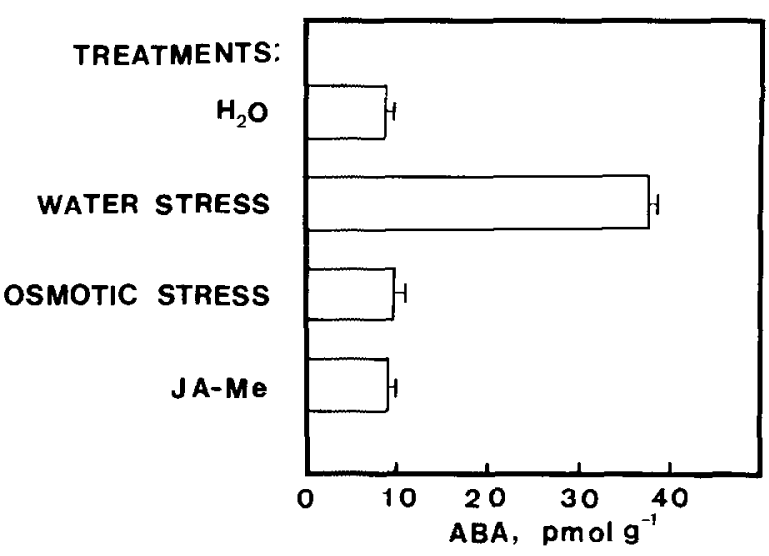

Fig. 3. Effects of water stress, osmotic stress and jasmonic acid methyl ester (JA-Me) on the levels of ABA in excised rice leaves. Excised rice leaves were treated with either water, $0.6 M$ sorbitol, $45 \mu M \mathrm{JA}-\mathrm{Me}$ or water stress in the light for $8 \mathrm{~h}$. Bars represent SE; 3 replicates.

leaves in the absence of sorbitol and JA-Me. However, both $\mathrm{D}$-arginine and $\alpha$-methylornithine decreased the levels of putrescine induced by osmotic stress or JA-Me, indicating that osmotic stress and JA-Me may affect the activities of both arginine decarboxylase and ornithine decarboxylase, enzymes responsible for the biosynthesis of putrescine [7]. Since D-arginine is more pronounced than $\alpha$-methylornithine in reducing the accumulation of putrescine induced by osmotic stress or JA-Me, arginine decarboxylase seems to be the major enzyme responsible for the accumulation of putrescine induced by osmotic stress or JA-Me.

Proline is known to be originated from L-glutamic acid, L-arginine and L-ornithine [19]. When rice leaf segments were treated with L-arginine and L-ornithine, proline level was significantly increased (Figure 4). It seems that proline and putrescine may share $\mathrm{L}$-arginine and $\mathrm{L}$-ornithine as precursors in excised rice leaves (Figure 5). Table 1 shows that both $\mathrm{D}$-arginine and $\alpha$-methylornithine resulted in an increase of proline levels in osmotically stressed and JA-Me-treated excised rice leaves. These results suggest that $\mathrm{D}$-arginine and $\alpha$-methylornithine result in more L-arginine and L-ornithine, respectively, available for proline accumulation.

\section{Discussion}

Sorbitol is a commonly used water stress agent.
Table 1. Effects of D-arginine and $\alpha$-methylornithine in the absence or presence of jasmonic acid methyl ester (JA-Me) or sorbitol on the levels of putrescine and proline in excised rice leaves

\begin{tabular}{lll}
\hline Treatment & $\begin{array}{l}\text { Putrescine } \\
\left(\mathrm{nmol} \mathrm{g}^{-1}\right)\end{array}$ & $\begin{array}{l}\text { Proline } \\
\left(\mu \mathrm{mol} \mathrm{g}^{-1}\right)\end{array}$ \\
\hline Water & $380 \pm 10$ & $0.25 \pm 0.02$ \\
D-Argine & $284 \pm 36$ & $0.25 \pm 0.01$ \\
$\alpha$-Methylornithine & $408 \pm 27$ & $0.25 \pm 0.01$ \\
JA-Me & $690 \pm 10$ & $0.40 \pm 0.01$ \\
JA-Me + D-Arginine & $520 \pm 6$ & $0.58 \pm 0.03$ \\
JA-Me + $\alpha$-Methylornithine & $576 \pm 4$ & $0.65 \pm 0.01$ \\
Sorbital & $930 \pm 58$ & $0.45 \pm 0.01$ \\
Sorbital + D-Arginine & $562 \pm 26$ & $0.60 \pm 0.01$ \\
Sorbital + $\alpha$-Methylornithine & $752 \pm 43$ & $0.72 \pm 0.02$
\end{tabular}

Excised rice leaves were incubated in the light for $8 \mathrm{~h}$ in D-arginine $(5 \mathrm{~m} M)$ or $\alpha$-methylornithine $(5 \mathrm{~m} M)$ in the absence or presence of JA-Me $(45 \mu M)$ or sorbitol $(0.6 M)$ (Mean \pm SE, 3 replicates).

The results of the present investigation show that osmotic stress and water stress affect differentially the levels of putrescine and proline in excised rice leaves. Putrescine accumulation is induced by osmotic stress, whereas proline accumulation is induced by water stress.

It has been reported that ABA accumulation is not required for proline accumulation in wilted barley leaves [20]. The present investigation demonstrated that water stress and ABA treatment behaved similarly in terms of accumulation of proline and putrescine in rice leaves. It seems that proline accumulation induced by water stress is most likely mediated through ABA in excised rice leaves. This suggestion is further supported by the observation that water stress resulted in an increase of endogenous ABA in excised rice leaves.

Contrary to water stress, sorbitol and JA-Me treatments do not result in an increase in endogenous $\triangle \mathrm{BA}$ levels in excised rice leaves. However, Larsson et al. [13] detected an increase in ABA levels in wheat exposed to osmotic stress by treatment with up to $20 \%$ polyethylene glycol. It is known that polyethylene glycol does not enter the cell wall space [18] and sorbitol does [8]. Whether different effects of sorbitol and polyethylene glycol on ABA levels are due to the different properties of two osmotica or different plant materials used is deserving of critical experimentation.

The present investigation shows that osmotic stress mimics the effects of JA-Me on the levels of putrescine and proline in excised rice leaves. At 


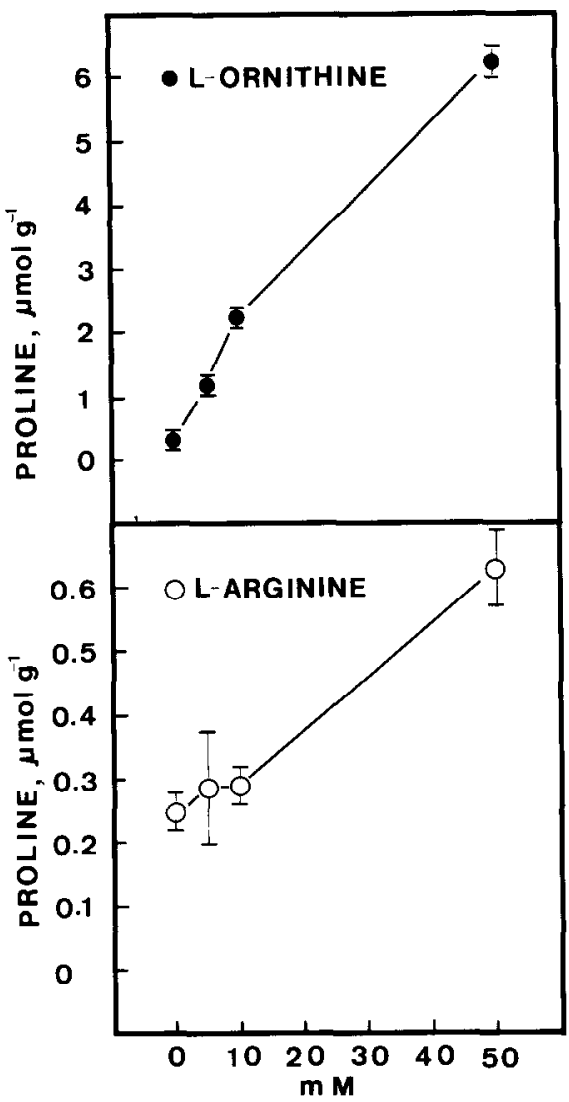

Fig. 4. Effects of L-arginine and L-ornithine on the levels of proline in excised rice leaves. Excised rice leaves were treated with various concentrations of $\mathrm{L}$-arginine or $\mathrm{L}$-ornithine in the light for $8 \mathrm{~h}$. Bars represents SE; 4 replicates.

present, we have technical problems in assaying endogenous jasmonates and are unable to provide direct evidence that osmotic stress would result in an increase in jasmonates in excised rice leaves. However, Parthier et al. [16] demonstrated that endogenous jasmonates increased in barley leaf segments exposed to $1 M$ sorbitol. If sorbitol treatment behaves similarly in excised rice leaves, then the effects of sorbitol on the levels of putrescine are more likely mediated by changes of endogenous jasmonates. Overall, our results suggest that the increase in proline and putrescine during stress are mediated by different mechanisms.

Of particular interest of the present investigation is the finding that proline levels increase when putrescine levels induced by osmotic stress and JA$\mathrm{Me}$ are reduced by $\mathrm{D}$-arginine and $\alpha$-methylornithine. We also observed that L-arginine and L-

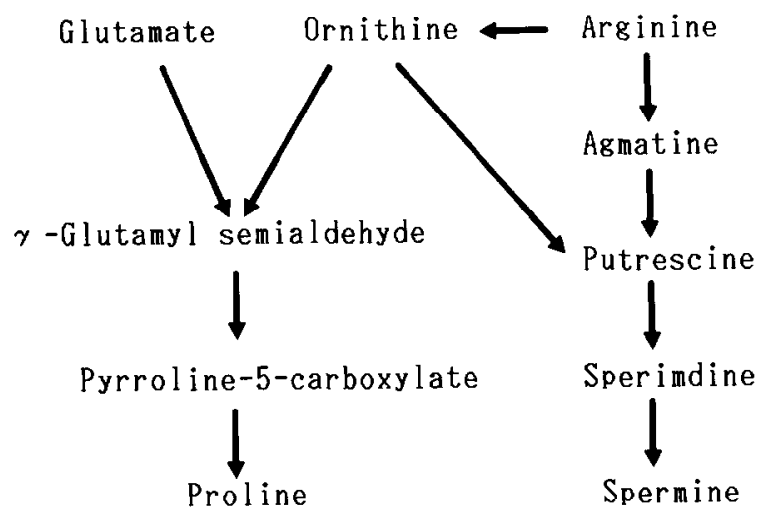

Fig. 5. Biosynthetic pathways of L-proline and polyamines in plant systems.

ornithine applied exogenously resulted in an increase of proline, suggesting that proline and putrescine may share L-arginine and L-ornithine as precursors in rice leaves. It seems that $\mathrm{L}$-arginine and L-ornithine are preferentially utilized as precursors for putrescine accumulation in excised rice leaves exposed to osmotic stress and JA-Me, and for proline accumulation in excised rice leaves exposed to water stress and ABA. However, this conclusion will not be firmly established until putrescine and proline accumulation during stress is followed upon labeling with $\mathrm{L}-\left[{ }^{14} \mathrm{C}\right]$ arginine or $\mathrm{L}-\left[{ }^{14} \mathrm{C}\right]$ ornithine.

\section{Acknowledgements}

This work was supported by a grant from the National Science Council, Republic of China (NSC 82-0409-B002-030). The assistance of Dr. Huu-Sheng Lur with ABA assay is gratefully acknowledged.

\section{References}

1. Aspinall D and Paleg LG (1981) Proline accumulation: physiological aspects. In: Paleg LG and Aspinall D (eds) The Physiology and Biochemistry of Drought Resistance in Plants, pp 280-295. Academic press: Sydney

2. Bates LS, Waldren RP, Tearc ID (1973) Rapid determination of free proline for water stress studies. Plant Soil 39: 205-207

3. Chen CT and Kao CH (1990) Comparative study of the metabolism of 1-aminocyclopropane-1-carboxylic acid and senescence of water-stressed and ABA-treated excised rice leaves. Plant Cell Physiol 31: 463-468 
4. Chen CT and Kao CH (1991) Senescence of rice leaves XXX. Levels of endogenous polyamines and dark-induced senescence of rice leaves. Plant Cell Physiol 32: 935-941

5. Chou IT, Chen CT, Kao CH (1991) Characteristics of the induction proline by abscisic acid and isobutyric acid in detached rice leaves. Plant Cell Physiol 32: 269-272

6. Ebenhardt HJ and Wegmann K (1989) Effects of abscisic acid and proline on adaptation of tobacco callus cultures to salinity and osmotic shock. Physiol Plant 76: 283288

7. Evans PT and Malmberg RL (1989) Do polyamines have roles in plant development? Annu Rev Plant Physiol Plant Mol Biol 40: 235-269

8. Flores HE and Galston AW (1982) Polyamines and plant stress: activation of putrescine biosynthesis by osmotic shock. Science 217: 1259-1261

9. Flores HE and Galston AW (1984) Osmotic stress-induced polyamine accumulation in cereal leaves I. Physiological parameters of the response. Plant Physiol 75: 102-109

10. Flores HE and Galston AW (1984) Osmotic stress-induced polyamine accumulation in cereal leaves II. Relation to amino acid pools. Plant Physiol 75: 110-113

11. Kandpal RP and Rao NA (1985) Changes in the levels of polyamines in ragi secdlings during water stress. Biochem Int 11: 365-370

12. Kao CH (1980) Senescence of rice leaves IV. Influence of benzyladenine on chlorophyll degradation. Plant Cell Physiol 21: $1255-1262$

13. Larsson $\mathbf{M}$, Larsson CM, Whitford PN, Clarkson DT (1989) Influence of osmotic stress on nitrate reductase activity in wheat (Triticum aestivum L.) and the role of abscisic acid. J Exp Bot 40: 1265-1271

14. Mousdale DMA and Knee M (1979) Poly-N-vinylpyrrolidone column chromatography of plant hormones with methanol as eluent. J Chromatogr 177: $398-400$
15. Norman SM, Poling SM, Maier VP (1988) An indirect enzyme-linked immunosorbent assay for $(+)$-abscisic acid in Citrus, Ricinus, and Xanthium leaves. J Agric Food Chem 36: 225-231

16. Parthier B (1991) Jasmonates, new regulators of plant growth and development: many facts and few hypotheses on their actions. Bot Acta 104: 446-454

17. Pesci $P$ and Reggiani $R$ (1992) The process of abscisic acidinduced proline accumulation and the levels of polyamines and quatenary ammonium compounds in hydrated barley leaves. Physiol Plant 84: 134-139

18. Rubinstein B (1982) Regulation of $\mathbf{H}^{+}$excretion. I. Effects of osmotic shock. Plant Physiol 69: 939-944

19. Stewart CR and Boggess SF (1977) The effect of wilting on the conversion of arginine, ornithine, and glutamate to proline in bean leaves. Plant Sci Lett 8: 147-153

20. Stewart CR and Voetberg G (1987) Abscisic acid accumulation is not required for proline accumulation in wilted leaves. Plant Physiol 83: 747-749

21. Turner I.B and Stewart GR (1986) The effect of water stress upon polyamine levels in barley (Hordeum vulgare L.) leaves. J Exp Bot 37: 170-177

22. Turner L and Stewart GR (1988) Factors affecting polyamine accumulation in barley (Hordeum vulgare L.) leaf sections during osmotic stress. J Exp Bot 39: 11-316

23. Walker-Simmons M (1987) ABA levels and sensitivity in developing embryos of sprouting resistant and susceptible cultivars. Plant Physiol 84: 61-66

24. Walton DC (1980) Biochemistry and physiology of abscisic acid. Annu Rev Plant Physiol 31: 453-489

25. Weiler EW (1980) Radioimmunoassays for the differential and direct analysis of free and conjugated abscisic acid in plant extracts. Planta 148: 262-272 